\title{
IMPLEMENTACIÓN DE UN PROGRAMA DE DIFUSIÓN DE LA FÍSICA EN ESTUDIANTES DEL NIVEL SECUNDARIO DE LA UNIDAD DE GESTIÓN EDUCATIVA LOCAL (UGEL) - TACNA
}

Responsable:Msc. José Antonio Tiburcio Moreno Miembro: Lic. Jesús Alfredo Chacaltana García

\section{RESUMEN}

La Física, además de ser una fascinante actividad, se dedica a estudiar los problemas fundamentales de la naturaleza; por ello es base de las demás ciencias y de las aplicaciones tecnológicas. Asimismo, nos ayuda a comprender, predecir, controlar, y muchas veces, a modificar el curso de los fenómenos. En muchos casos, la Física se enseña en las instituciones educativas en forma descontextualizada. Por eso a los estudiantes les parece tan lejana de su vida cotidiana Es en este marco que planteamos nuestro Proyecto de difusión de la Física a estudiantes del nivel secundario para que se interesen en estudiar estos fenómenos que han sido de vital importancia en el desarrollo de nuestra sociedad

\begin{abstract}
Physics, besides being a fascinating activity, is dedicated to exploring the fundamental problems of nature, so is the basis of other sciences and technological applications. It also helps us to understand, predict, control, and often, to alter the course of events. In many cases, the physics is taught in educational institutions in the form off context, that's why the students they seem so distant from their daily lives is this context that we raise our project of dissemination of physics to students at the secondary level for the interested in studying these phenomena have been of vital interest in the development of our society
\end{abstract}

\section{INTRODUCCIÓN}

La Física es la ciencia que estudia los fenómenos de la naturaleza. Según esta definición general, se engloba a otras ciencias como la astronomia, la química, la geología, etc. La Física propiamente dicha estudia las propiedades de los cuerpos y los fenómenos o cambios accidentales producidos en ellos por los agentes naturales.

Tradicionalmente, y más por razones pedagógicas que de otra indole, se han construido cinco grandes ramas de esta ciencia: mecánica, electromagnetismo, termodinámica, acústica y óptica.

A estas ramas corresponden el estudio de movimiento de los cuerpos y sistemas, la electricidad y el magnetismo, la transmisión del calor, el sonido y las perturbaciones en los gases, y la luz en su naturaleza y propagación. Actualmente, debido al avance en el estudio de la naturaleza del mundo de lo infinitamente pequeño, han surgido nuevas ramas, como son la física nuclear, la física atómica, y la mecánica cuántica.

La Física desempeña un papel decisivo en la cultura moderna. Su desarrollo ha contribuido al progreso de muchas otras actividades humanas, de la medicina a los viajes espaciales, de la economia a las telecomunicaciones, etc. No es posible tener una educación moderna sin comprender algunas ideas y hechos del terreno de la Física. Esta ciencia es la que ha permitido el desarrollo de la telegrafía y la telefonía y la que nos permite ver en la televisión lugares distantes. La Física es el fundamento de la generación de la electricidad; ha hecho posible enviar al hombre a la Luna, diseñar y construir nuevos aviones, fabricar grandes y pequeñas computadoras, explotar y aprovechar las fuentes de energía que tanta importancia económica y política tienen en la actualidad, etc.

Asimismo, la Física ha contribuido de manera decisiva al desarrollo tecnológico, pero no es menos cierto que la tecnologia ha dado a la Física poderosas herramientas de trabajo que necesita esta para su continua evolución. Este continuo inquirir en la naturaleza nos permite profundizar cada vez más y alcanzar niveles de comprensión cada vez mejores en un proceso inacabable.

En la actualidad se trabaja para unificar las cuatro interacciones fundamentales conocidas: la gravitatoria, la débil, la fuerte y la electromagnética. Si bien estas tres últimas han permitido realizar enormes avances en cuanto a la unificación, la primera aún resiste este 
proceso, que intenta cerrarse mediante las teorias de gran unificación y, más recientemente, la llamada teoria de supercuerdas.

Con el desarrollo de las diversas ramas, la Física ha adquirido una estructura que facilita el estudio sistemático. No debe creerse, sin embargo, que esta estructura se mantiene inalterada: a la luz de los nuevos hechos experimentales y de los avances teóricos surgen nuevas áreas, se funden unas con otras y cambian la relación entre ellas.

$A$ veces la Física se enseña en las instituciones educativas fuera de un contexto real. Por ello a las personas, y generalmente a los estudiantes, les parece tan lejana de su vida cotidiana. Todo lo que hay que intentar hacer es una conexión entre la comprensión de la Física y las preguntas que todos escuchamos sobre el mundo en que estamos viviendo.

Es responsabilidad de los educadores y de los científicos aclarar estas inquietudes con explicaciones que cualquier persona pueda comprender.

Uno de los grandes desafíos de los profesores es tomar conciencia de que tienen que estimular la curiosidad de sus alumnos y que lo deben hacer con la ayuda de los científicos, siendo curiosos ellos mismos también, no perdiendo el espíritu inquieto, así podrán conducir el entusiasmo de los estudiantes. Si no, se transformarán en robots, en personas sin interés por el mundo, sólo haciendo sus vidas de manera automática.

Una vez que nos damos cuenta de que la Física es en realidad fundamental, entonces tenemos un marco para comprender muchas cosas que suceden en la vida, por lo que, si un objeto disminuye su velocidad, podemos decir "Tal vez exista una fuerza que cambie el movimiento de ese objeto". Si la velocidad no cambia, no hay fuerza. $Y$ esta es una idea básica, pero tomó a las sociedades milenios para descubrirla. Por tanto, incluso ideas tan simples en Física no siempre son comprendidas por el público general. La Física Cuántica es todavia más dificil porque está más alejada de la intuición diaria.

Esas ideas son dificiles. Pero ser curioso del mundo es descubrir que tras la apariencia de todos los dias hay reglas básicas que dan forma al mundo. Esto es muy potente y no es tarea de la gente descubrirlo, deben ser guiados. Y éste es, una vez más, el rol de los profesores.

En en este trabajo, se trata de comunicar qué sucede hoy en la investigación, por qué se estudia lo que se estudia, qué tan importante para la sociedad es comprender esa clase de cosas. Hacer avanzar el conocimiento es el rol de los científicos, pero también es su responsabilidad hacer accesible ese conocimiento a todos. $Y$ creemos que es importante para la generación joven comprender que la Física es una dimensión muy interesante donde practicar cosas, es muy igualitaria.

Es en este marco que planteamos nuestro Proyecto de difusión de la Física a estudiantes del nivel secundario para que se interesen en estudiar estos fenómenos que han sido de vital importancia en el desarrollo de nuestra sociedad, tal es así que en este trabajo planteamos las siguientes estrategias para la promoción de iniciativas para mejorar la enseñanza de la Física en el nivel secundario:

1. Jornada de trabajo en los laboratorios de la Facultad de Ciencias, para los estudiantes del nivel secundario. A través de estas jornadas se pretende motivar a los estudiantes hacia el trabajo experimental, contribuir a mejorar la comprensión de fenómenos físicos mediante la realización de experiencias de laboratorio adaptadas a los contenidos del nivel secundario y fomentar la colaboración entre los profesores de secundaria y universidad mediante la preparación conjunta de las sesiones.

2. Llevar a cabo la Tarea de El Armario de Prácticas, en el cual se acondicionarán maletas o armarios móviles con material experimental necesario y manuales de experimentación detallados para realizar demostraciones de laboratorio de cursos de Física en las aulas de las instituciones educativas en el caso que no puedan realizar los experimentos del acápite anterior, asimismo desarrollar los instrumentos de evaluación para tales casos.

3.Participación en Jornadas de Didáctica, en las cuales se intercambiaran experiencias de innovación educativa, interacción con otras áreas (Química, Biología, Matemáticas) y Presentación de las actividades de estas jornadas

4.Implementación de talleres prácticos a través de demostraciones sencillas, cualitativas $y / 0$ cuantitativas, con material accesible o reciclable y que permitan analizar aspectos básicos de la Fisica.

5.Participación activa en la Semana de la Física, organizada por el Departamento de Física de la Facultad de Ciencias de la Universidad Nacional Jorge Basadre Grohmann. 
6.Participación con exponentes estudiantiles de los grupos formados, en el Concurso Regional de Física organizado anualmente por el Departamento Académico de Física

Antes de llevar a cabo las actividades planteadas en este proyecto, se aplicó una prueba de aptitud vocacional en instituciones educativas de la UGEL Tacna, seleccionadas para tal fin: Coronel Francisco Bolognesi (Varones), Alexander Von Humboldt (Mixto), Corazón de María(Femenino), Marcelino Champagnat (Varones).

La razón por la cual se tomaron las muestras en estas instituciones educativas fueron la disposición de apoyo para este trabajo y además, debido a la huelga magisterial y de los docentes universitarios, se restringió el trabajo en otras instituciones educativas.

Para analizar las preferencias vocacionales en los grupos de alumnos se tomaron en cuenta los siguientes intereses:

IM - Intereses Mecánicos

TEI - Intereses en la Técnica, Electrónica e Ingeniería

ITAL - Intereses para el trabajo al Aire Libre:

IC - Intereses Cientificos:

CCC - Intereses en Cómputo, Cuentas y Cálculo:

NRV - Intereses en Negocios, Relaciones y Ventas:

DSE - Intereses en Dirección, Supervisión y Ejecutivos.

EO - Intereses en Empleos de Oficina.

SS - Intereses en Servicios Sociales.

IL - Intereses Literarios:

AMR - Intereses Artísticos, Musicales y Recreación. (Arte y/o Música)

Los resultados para una muestra de 32 estudiantes de la Institución Educativa Corazón de María de las secciones de quinto grado de educación secundaria, los resultados se muestran en la Figura 1

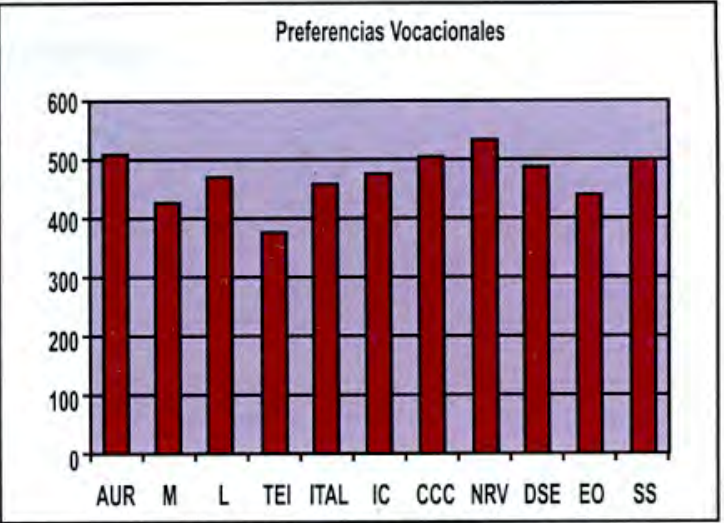

Figura 1: Preferencias vocacionales I.E.

Corazón de María.

Se observa de la Figura anterior que la primera opción de preferencias vocacionales para el caso de la Institución Educativa Corazón de Maria es la correspondiente a intereses en Negocios, Relaciones y Ventas (NRV); los altos puntajes en esta área indican una inclinación hacia trabajos en los cuales se hace uso de la persuasión y el contacto humano, tales como ventas, el mercadeo y la política para las cuales se requiere un alto grado de aptitud verbal.

Como puede apreciarse, de los resultados obtenidos en la encuesta, los intereses científicos y/o tecnológicos no son de mucho interés para el caso de las alumnas de la Institución Educativa Corazón de María; en el cuestionario que se les aplicó se consideró la posibilidad de agrado o desagrado de estudiar una carrera de ciencias, siendo el indicador prácticamente nulo en este caso.

Analizando esta situación, pudimos apreciar que las docentes encargadas de los cursos de ciencias no son especialistas en la materia y toda la programación de estos cursos es de manera teórica tradicional sin considerar experiencias de laboratorio y de campo, lo que indica manifiestamente la falta de interés en el campo de las ciencias en los intereses vocacionales de las alumnas.

Se realizó el estudio de la Institución Educativa Particular Alexander Von Humboldt, la cual es una Institución Educativa mixta; el análisis de intereses vocacionales se realizo para los estudiantes varones y mujeres por separado, los resultados se muestran en la Figura 2: 


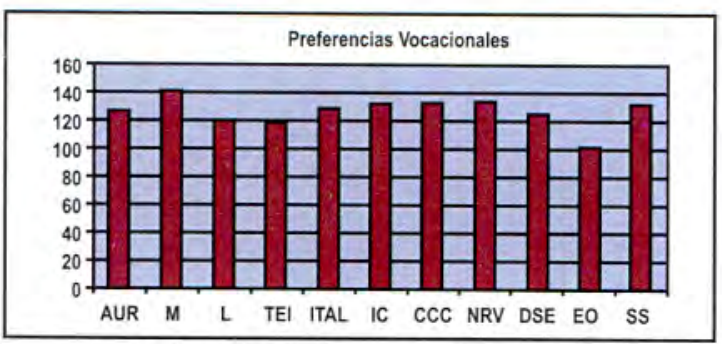

Figura 2. Institución Educativa Particular Alexander Von Humboldt (Varones)

Puede apreciarse el interés vocacional para el caso de los varones está ligado a Intereses Mecánicos (IM); los altos puntajes en estos intereses demuestras una fuerte atracción por trabajos relacionados con la Mecánica y reparación de equipos. Las profesiones relacionadas en este campo serian: Ingeniería Mecánica, Ingeniería Sanitaria, Ingeniería Industrial, Ingeniería de Materiales, Ingeniería Metalúrgica, Electromecánica, Física y ramas afines a las anteriores.

Debemos mencionar que en esta Institución Educativa el curso de Física se programa a partir del cuarto grado de educación secundaria, materia que está asumida por un docente especialista, adecuando los temas tratados con material de laboratorio y de campo.

Para el caso de estudiantes mujeres en esta Institución Educativa, los resultados son mostrados en la Figura 3 :

Puede apreciarse el interés vocacional para este caso está ligado a Intereses Artísticos, Musicales y Recreación. (Arte y/o Música) (AMR). Un alto puntaje en esta área indica que existe un fuerte interés por los campos creativos del arte, la música o el teatro y que tienen originalidad e imaginación.

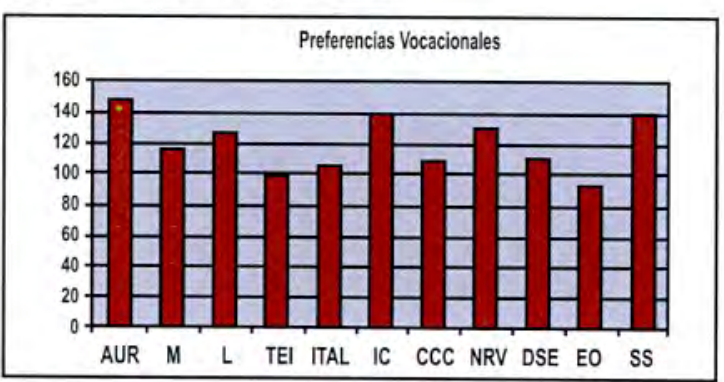

Figura 3. Institución Educativa Alexander Von Humboldt (Mujeres)

Del análisis de las Figuras 2 y 3 , podemos concluir que los intereses o preferencias vocacionales para varones y damas en la Institución Educativa Particular Alexander Von Humboldt, son relativamente diferentes. Aunque se da mucho énfasis en los cursos de Ciencias en el Plan de Estudios de esta Institución Educativa, no hay un marcado interés en la preferencia para el estudio de las ciencias.
Para el caso de la Institución Educativa Francisco Bolognesi, que es una Institución Educativa de varones, los resultados se muestran en la Figura 4:

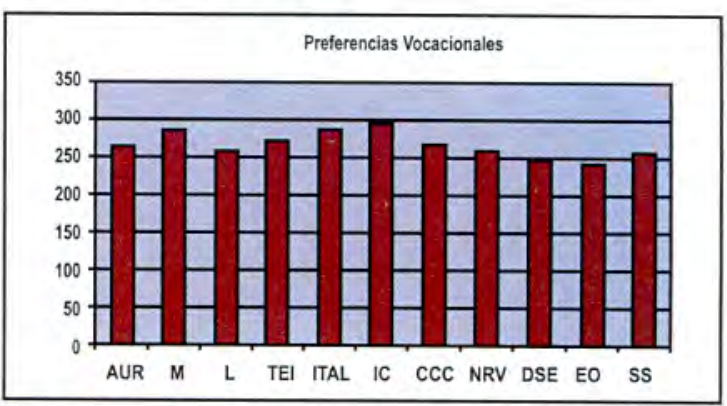

Figura 4. Institución Educativa Francisco Bolognesi

Se puede apreciar que los intereses vocacionales en este caso están ligados a Intereses Cientificos (IC), en los cuales hay alto interés hacia la investigación y trabajos de laboratorio, así como en los aspectos teoricos de la Fisica y en los porcesos de sistematizacion de datos.

El análisis demuestra que en esta Institución Educativa hay un marcado interés en lo que concierne al estudio de las ciencias especialmente la física, química, matemáticas, lo cual ha quedado demostrado por la participación brillante de esta Institución Educativa que ha obtenido primeros lugares en las olimpiadas de Física y Matemáticas en años recientes.

Se realizó el estudio en la Institución Educativa Champagnat, cuyos resultados se muestran en la Figura 5:

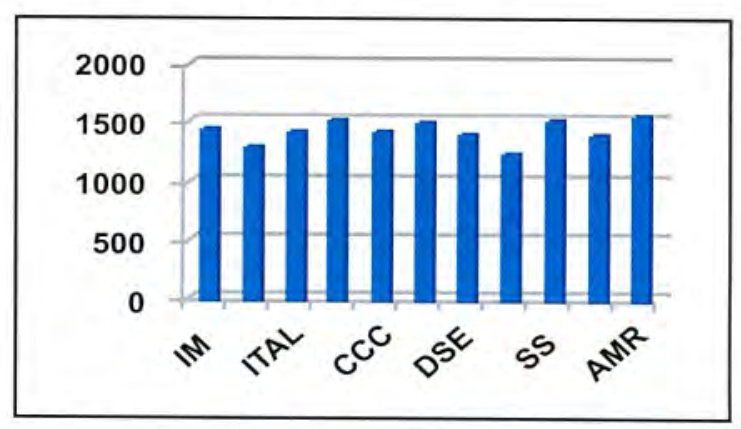

Figura 5. Institución Educativa Champagnat

Se observa que los interés vocaciones en esta Institución Educativa están relacionados a aspectos: Artísticos, Musicales y Recreación. (Arte y/o Música) (AMR), y como segunda opción a Intereses Científicos (IC), debido fundamentalmente a la presencia de docentes especialistas que están encargados del dictado de las materias de ciencias.

\section{PROGRAMA DE DIFUSIÓN DE LA FÍSICA}

En este contexto es que nos hemos dedicado a incentivar el estudio de la Física, por ser una 
disciplina que es uno de los pilares en la generación de nuevas tecnologias que nuestro pais requiere. Lamentablemente no hay mucho apoyo por parte de los profesores comprometidos en la enseñanza de las ciencias y por falta de motivación en el aprendizaje de las ciencias de manera divertida y sin traumas.

El Departamento de Física y la Escuela de Fisica Aplicada han realizado distinto talleres de motivación para difundir el interés de los alumnos en seguir en el estudio de las ciencias, especialmente la Fisica, entre los cuales tenemos los siguientes: Curso de Actualización en Fisica para docentes del magisterio, en donde se abordó la problemática de la enseñanza de la fisica y el desarrollo de contenidos de los principales temas de los cursos de Física en la diferentes instituciones educativas.

Debemos indicar que los docentes del Departamento Académico de Física brindan apoyo y asesoramiento en la realización de trabajos experimentales que son presentados a la Feria Nacional de Ciencia y Tecnologia auspiciada por el Consejo Nacional de Ciencia y Tecnología

Asimismo, se programaron distintas visitas guiadas a los laboratorios de la Escuela de Física Aplicada, para incentivar el desarrollo de interrelación de la parte teórica y experimental y detallar analiticamente las principales aplicaciones de la Física en la vida diaria. Uno de los objetivos del grupo es apoyar todo tipo de actividades que sirvan para la promoción de la enseñanza de la Física, asi como para la difusión de la Ciencia en cualquier ámbito. Este compromiso nos ha llevado, por ejemplo, a participar en jornadas de debate sobre los nuevos itinerarios curriculares de ciencias en la educación secundaria, o a la organización de jornadas de demostración para profesores de bachillerato del empleo de las nuevas tecnologias audiovisuales. Desde nuestro punto de vista la valoración de las actividades realizadas hasta el momento es altamente positiva entre los destinatarios de las mismas.

\section{CONCLUSIONES}

1.Según todo lo expuesto podemos decir que los jóvenes pierden interés en la ciencia debido a las caracteristicas del modelo educativo vigente y a la poca interaccion de los aspectos teóricos con las aplicaciones en la vida diaria.

2.Para revertir esta situación es fundamental desarrollar la capacidad de los profesores de transmitir no solo conocimientos, sino también una formación integral al alumno. Además, se necesita un cambio de fondo en las condiciones laborales de los docentes e investigadores, ya que hasta entonces el alumno no vislumbrará condiciones favorables para su futuro profesional.

3.Por último, todos deberiamos comprometernos para crear una cultura que privilegie la búsqueda del conocimiento a traves del desarrollo de la ciencia, la investigación y la perseverancia; ya que el cambio en la sociedad solo es posible con la participación de todos.

\section{RECOMENDACIONES}

\section{Enseñanza de la Física con Tecnologia}

El proyecto Enseñanza de Fisica con Tecnología (EFIT) incorpora el uso de Tecnologías de la Información y Comunicación (TIC) en escuelas secundarias. A continuación exponemos las recomendaciones que deberían ser implementadas para lograr mayor impacto en la enseñanza de la Física en Instituciones Educativas del nivel secundario:

- Incorporar sistemática y gradualmente el uso de las tecnologías de la información y la comunicación (TIC) en la escuela secundaria para la enseñanza de la Física.

- Poner en práctica el uso significativo de las TIC basándose en un modelo pedagógico orientado a mejorar y a enriquecer el aprendizaje de los contenidos curriculares.

- Explorar el uso de las TIC para la enseñanza de contenidos más allá del currículo, con base en el acceso a ideas importantes en ciencias.

- Estar relacionadas con un área específica de la física escolar.

- Contar con representaciones ejecutables de objetos, conceptos y fenómenos de la ciencia.

- Permitir un tratamiento fenomenológico de los conceptos científicos.

- Se útiles para abordar situaciones que no pueden estudiarse con los medios tradicionales de enseñanza.

\section{BIBLIOGRAFÍA}

Rojano, T., "Incorporación de entornos tecnológicos de aprendizaje a la cultura escolar: proyecto de innovación educativa en matemáticas y ciencias en escuelas secundarias públicas de México", Revista Iberoamericana de Educación. Número 33, pp 135-165, Septiembre-Diciembre 2003.

Tonda Mazon, J. Enseñanza de la Fisica con Tecnologia, Editorial Reverté, Madrid, 1998.

Gvirtz, S., "Hacia un sistema educativo justo, democrático y de calidad: construyendo un futuro para la Argentina del siglo XXI" sobre el tema ¿Cómo revertir la crisis educativa argentina?, Academia Nacional de Educación, 2004. 


\section{ANEXO}

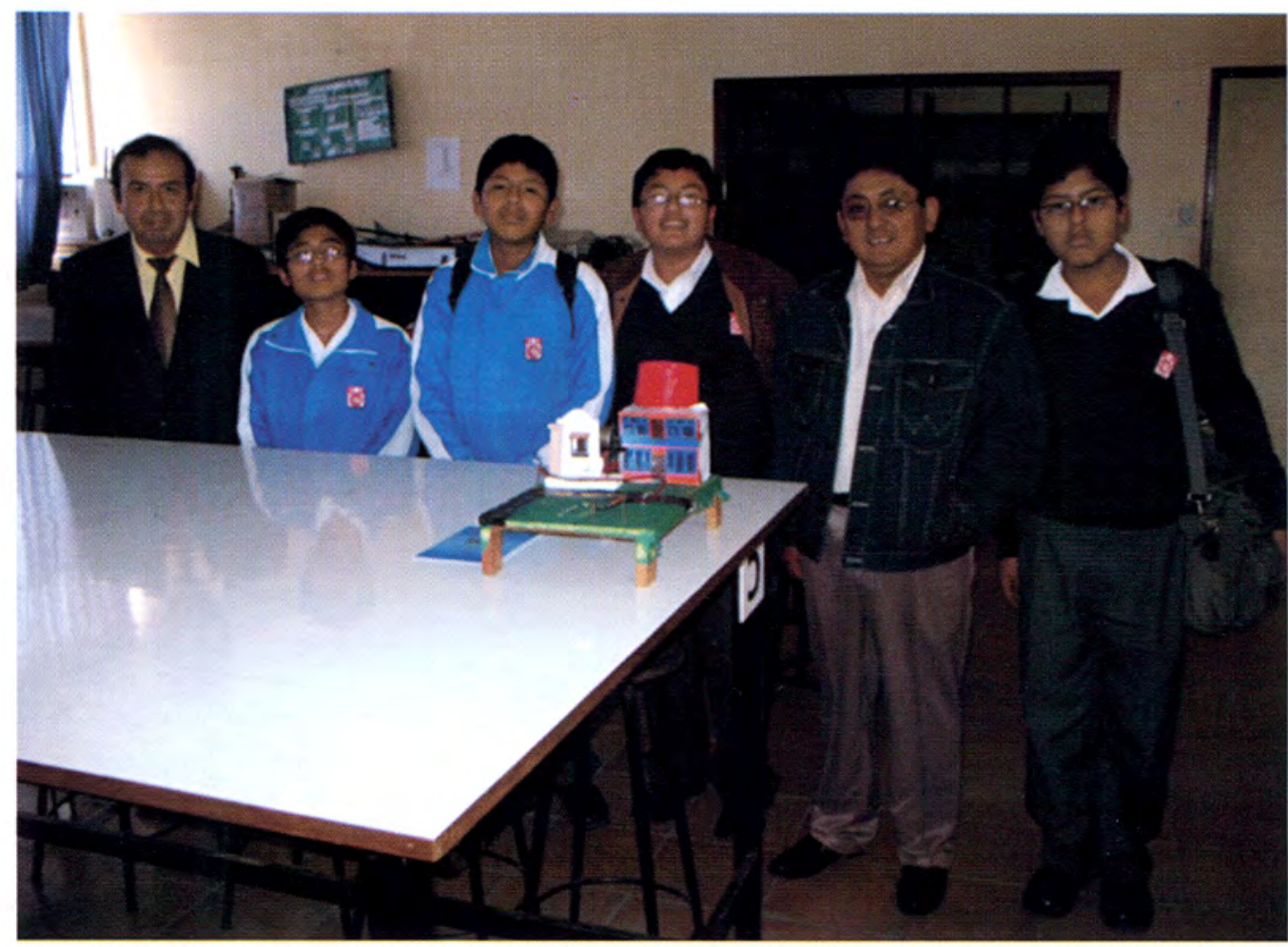

Foto 1: Responsable del proyecto: asesorando un proyecto, de ciencias a los estudiantes de la Institución Educativa Coronel Bolognesi .

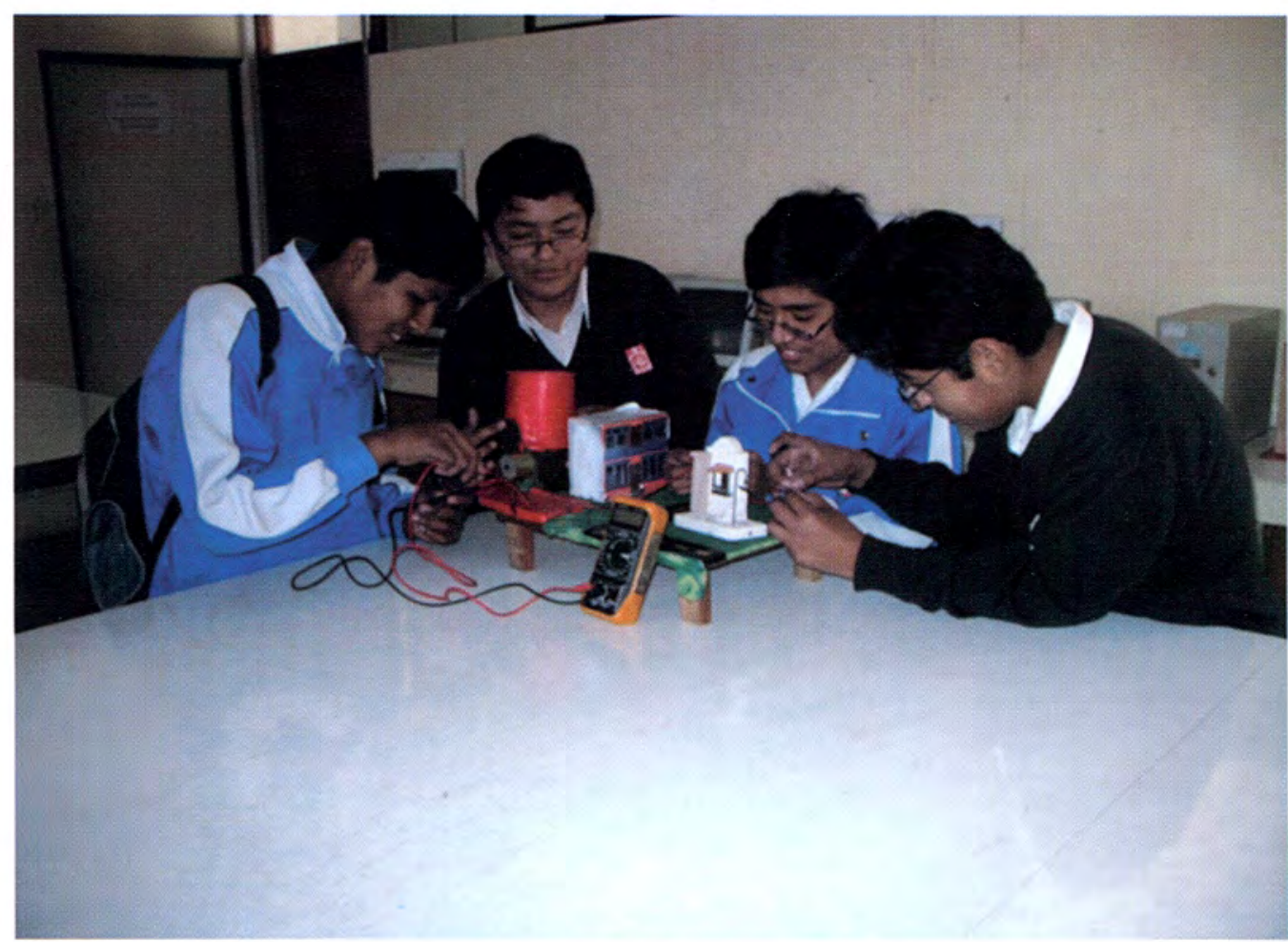

Foto 2: Estudiantes de la Institución Educativa Bolognesi, realizando experimentos en el laboratorio de electricidad y electronica de la Universidad Nacional Jorge Basadre Grohmann 